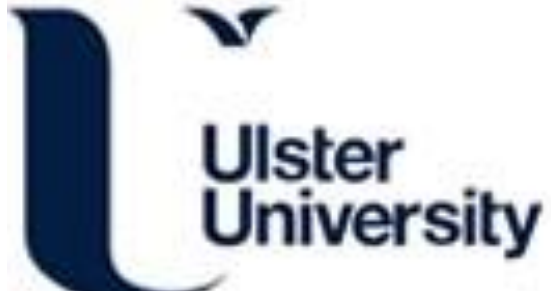

A Drift Diffusion Model of Biological Source Seeking for Mobile Robots

Rano, I., Khamassi, M., \& Wong-Lin, K. (2017). A Drift Diffusion Model of Biological Source Seeking for Mobile Robots. In ICRA 2017 - IEEE International Conference on Robotics and Automation (pp. 3525-3531). [7989403] IEEE. https://doi.org/10.1109/ICRA.2017.7989403

Link to publication record in Ulster University Research Portal

Published in:

ICRA 2017 - IEEE International Conference on Robotics and Automation

Publication Status:

Published (in print/issue): 21/07/2017

DOI:

10.1109/ICRA.2017.7989403

Document Version

Author Accepted version

\section{General rights}

Copyright for the publications made accessible via Ulster University's Research Portal is retained by the author(s) and / or other copyright owners and it is a condition of accessing these publications that users recognise and abide by the legal requirements associated with these rights.

\section{Take down policy}

The Research Portal is Ulster University's institutional repository that provides access to Ulster's research outputs. Every effort has been made to ensure that content in the Research Portal does not infringe any person's rights, or applicable UK laws. If you discover content in the Research Portal that you believe breaches copyright or violates any law, please contact pure-support@ulster.ac.uk. 


\title{
A Drift Diffusion Model of Biological Source Seeking for Mobile Robots
}

\author{
I. Rañó ${ }^{1}$, M. Khamassi ${ }^{2}$ and K. Wong-Lin ${ }^{1}$ \\ ${ }^{1}$ Intelligent Systems Research Centre, Ulster University, Northern \\ Ireland, UK \\ ${ }^{2}$ Institut des Systèmes Intelligents et de Robotique, Université \\ Pierre et Marie Curie, France
}

(C) 20xx IEEE. Personal use of this material is permitted. Permission from IEEE must be obtained for all other uses, in any current or future media, including reprinting/republishing this material for advertising or promotional purposes, creating new collective works, for resale or redistribution to servers or lists, or reuse of any copyrighted component of this work in other works. 


\title{
A Drift Diffusion Model of Biological Source Seeking for Mobile Robots
}

\author{
I. Rañó ${ }^{1}$ and M. Khamassi ${ }^{2}$ and K. Wong-Lin ${ }^{1}$
}

\begin{abstract}
Braitenberg vehicles have been used in multiple real world robotic implementations of bio-inspired local navigation. While sensor based control strategies - including existing models of Braitenberg vehicles - typically neglect sensor noise, real robot implementations suffer from different levels of noise, especially in the case of low cost robots and highly stochastic environments. This paper presents a novel drift-diffusion model of Braitenberg vehicle 3a - a bio-inspired source seeking controller for non-holonomic robots - accounting for the sensor noise. The stochastic differential equations obtained provide means to accurately simulate the behaviour of this bio-inspired control mechanism. Although these equations do not have analytic solutions in general, under some simplifying assumptions, we obtain the deterministic equations for the average and dispersion of the vehicles while performing source seeking. Moreover, we found an analytic bound for the distribution of the heading direction of the robots. Simulations illustrate and confirm the theoretical results presented.
\end{abstract}

\section{INTRODUCTION}

Braitenberg vehicles model simple stimulus driven animal movement towards (or away from) a stimulus [1]. Known in biology as taxes, these models qualitatively illustrate how such a set of controllers can become a basis for local bioinspired navigation [2]. Although the original formulation is purely sensor-based, a potential function can be defined on the environment to drive local navigation. In contrast, for instance, to potential-based methods in robotics, Braitenberg vehicles inherently account for the non-holonomic motion of animals regardless of their locomotive system [3]. The original qualitative models are highly intuitive, yet their mathematical formulation has been used to predict not so intuitive features of their behaviour like deterministic chaos [4]. Like many other navigation control techniques for mobile robots, these models assume a deterministic pose and neglect sensor noise in their mathematical formulation for simplification purposes. Assumptions of noiseless sensor readings might be unrealistic in many cases, such as swarm robotics and any low cost robot, but also when the environment is highly stochastic, for example in underwater robotics. This paper presents the first model of a Braitenberg vehicle $3 \mathrm{a}$ (see figure 1) in the presence of sensor noise as a stochastic differential equation. Drift diffusion models are widely used in physics and chemistry [5], decision-making [6], and biological movement [7], among others. As we will show later, the model enables realistic simulations, and

\footnotetext{
${ }^{1}$ Iñaki Rañó and KongFatt Wong-Lin are with the Intelligent Systems Research Centre, Ulster University, UK. e-mail: $\{$ i.rano, k. wong-lin\}@ulster.ac.uk

${ }^{2}$ Mehdi Khamassi is with the Institut des Systèmes Intelligents et de Robotique, Université Pierre et Marie Curie, France e-mail: mehdi.khamassi@upmc.fr
}

provides a better understanding of this bio-inspired control mechanism in real world conditions.

Because of their qualitative nature, the principles governing Braitenberg vehicles have been implemented in different ways in many successful robotic works. A robot imitating female cricket phonotaxis is presented in a series of works [8], [9], [10], where the goal of the robot was to move towards a sound source. A combination of vehicles $2 \mathrm{a}$ and $3 \mathrm{~b}$ was implemented using a spiking neural network, resulting in an excellent performance under quite adverse outdoor conditions. Contrary to other works and to the existing models of Braitenberg vehicles [2], the sensormotor connection is not functional but dynamic, due to the nature of the spiking neural networks used. Another implementation of phonotaxis is presented in [11] where the main contributions are implementing a model of the central auditory system of rats and integrated a pinnea. This work uses vehicle $3 \mathrm{a}$ to drive the robot motion towards the sound source. Further, a phonotaxic lizard robot is presented in [12] where a Braitenberg vehicle and a bang-bang controller are compared. This implementation works successfully across a wide range of frequencies for an empirically tuned vehicle type 2b. The works in [13] [4] present similar obstacle avoidance mechanisms using vehicle $2 \mathrm{~b}$ and with proximity sensors. The stimulus is an estimate of the free area around the robot, which can be shown to produce chaotic trajectories [4].

The behaviour of Braitenberg vehicles can be properly described by their deterministic model when the signal to noise ratio is high enough, as the noise can be neglected. However, they have been also used successfully in more adverse conditions. A pioneering work in odour localisation in robotics [14] analysed experimentally the behaviour of Braitenberg vehicles $3 \mathrm{a}$ and $3 \mathrm{~b}$. Based on symmetrically located chemical sensors this work shows how a robot can move towards high concentrations of chemicals in the environment and stay close to the odour sources. An implementation of fish rheotaxis is presented in [15], where pressure sensors on the sides of the fish robot imitating the fish lateral line were used to keep the orientation of the robotic fish aligned with a laminar flow. Although the variation in the lateral position of the robot using Braitenberg vehicle $2 \mathrm{~b}$ controller is larger than a PID controller, the fish heading direction results in a better alignment with the flow. Inspired by electric fish, [16] presents another underwater robotic application of Braitenberg vehicles, where a robot approaches conductive objects and avoids isolating ones in a pond. Grounded on the bilateral symmetry of animals, the steering control is driven by the current difference measured 
by electrodes on both sides of the robot, one of the principles of Braitenberg vehicles.

Through the literature we find multiple empirical applications of Braitenberg vehicles even in highly stochastic environments, and using robots with noisy sensors. While existing mathematical models work under ideal conditions, we will show it is not sufficient to understand the robot behaviour in realistic scenarios with noise. The contribution of this paper is threefold. First, we present the first stochastic model (as a drift-diffusion model) of this biologically inspired source seeking mechanism (Braitenberg vehicle 3a) under sensor uncertainty. The resulting stochastic differential equation allows realistic simulations of this movement mechanisms, and a subsequent analysis of the resulting simulated trajectories for different noise realisations. Second, under certain assumptions, the model can provide analytical results like the deterministic equations driving the average and dispersion evolution of the vehicles over time, and a bound for the angular dispersion. Finally, it has been shown recently that adding noise to navigation functions can improve the obstacle avoidance behaviour in the presence of potential minima [17]. However, little is known about the effect that this would have on the accuracy to reach the target position. This work gives the first steps in the direction to find what the effect of noise is for a non-holonomic target reaching controller in the presence of noise.

The rest of the paper is organised as follows. Section II presents the assumptions and develops the model of Braitenberg vehicle $3 \mathrm{a}$ as a stochastic differential equation. Section III shows that, under certain conditions, the deterministic equations of the evolution of the probabilistic properties of the model can be obtained in a simple form. Further simplifications allow finding the limit value of the variance of the vehicle heading direction. Section IV presents computer simulations of the stochastic and deterministic equations of Braitenberg vehicle $3 \mathrm{a}$ to illustrate the results. The paper concludes presenting some remarks and future lines of work in Section V.

\section{A DRIFT DIFFUSION MODEL OF BRAITENBERG VEHICLE 3A}

Before developing the drift-diffusion model of Braitenberg vehicle 3 a we briefly introduce its original qualitative model. As figure 1 shows, vehicle 3 a corresponds to a wheeled robot with two symmetrically arranged sensors connected in a decreasing way (inhibitory) to the wheel motor of the same side (ipsilateral connection). The decreasing connection, depicted by the sign '-' in the figure, means that high sensor stimulus produce slow wheel turning speeds, and the higher the stimulus the lower the speed. For instance, if the stimulus lies on the right side of the vehicle, the intensity perceived in the right sensor will be higher than the intensity perceived in the left one. Given the vehicle internal connections, the right wheel will turn slower than the left one, making the vehicle turn towards high values of the stimulus. The first mathematical model of this bio-inspired non-linear control mechanism for non-holonomic vehicles was presented in [18]

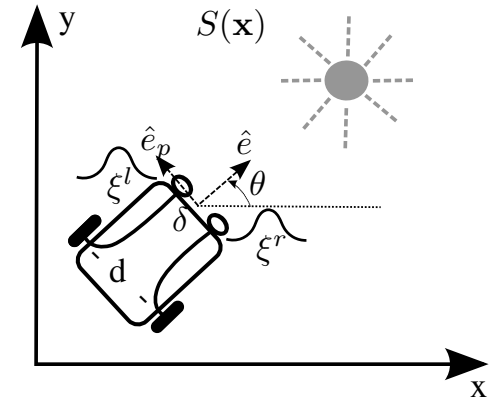

Fig. 1. Braitenberg vehicle 3a

assuming noiseless sensors. In this paper we introduce a white noise signal following a Gaussian distribution in the sensor readings (see figure 1) and develop the corresponding model which requires the use of stochastic systems theory.

Like in the case of deterministic Braitenberg vehicles [2] we will assume the vehicle is immersed in a deterministic two dimensional stimulus modelled as a smooth function of the point in the environment $S(\mathbf{x})$. We assume that the sensors present a noise term, $\xi_{t}$, with a variance depending on the stimulus perceived $\xi_{t}=\sigma(S(\mathbf{x})) d W_{t}$, where $W_{t}$ is a Wiener process. Therefore the noise at every point in time follows a zero mean Gaussian distribution. We assume the variance of the noise depends smoothly on the stimulus measured, i.e. $\sigma(s)$ is a smooth function of $s$. Obviously, because the vehicle has two sensors, left and right, there are two such noise processes we will denote by $\xi_{t}^{l}$ and $\xi_{t}^{r}$, but we assume both have identical statistical properties. The connection function $F(s)$ sets the speeds for each wheel according to the corresponding sum of the stimulus measured and the noise. Therefore the speed of the right and left wheels are:

$$
\begin{aligned}
& v_{r}=F\left(S\left(\mathbf{x}_{r}\right)+\xi_{t}^{r}\right) \\
& v_{l}=F\left(S\left(\mathbf{x}_{l}\right)+\xi_{t}^{l}\right)
\end{aligned}
$$

where $\mathbf{x}_{l}=\left[x_{l}, y_{l}\right]$, and $\mathbf{x}_{r}=\left[x_{r}, y_{r}\right]$ are the positions of the left and right sensors. Assuming $F(s)$ is also smooth enough we can approximate the wheel speeds as:

$$
\begin{aligned}
v_{r} & \approx F\left(S\left(\mathbf{x}_{r}\right)\right)+F^{\prime}\left(S\left(\mathbf{x}_{r}\right)\right) \sigma\left(S\left(\mathbf{x}_{r}\right)\right) d W_{t}^{r} \\
v_{l} & \approx F\left(S\left(\mathbf{x}_{l}\right)\right)+F^{\prime}\left(S\left(\mathbf{x}_{l}\right)\right) \sigma\left(S\left(\mathbf{x}_{l}\right)\right) d W_{t}^{l}
\end{aligned}
$$

where $d W_{t}^{r}$ and $d W_{t}^{l}$ are the stochastic processes driving the noise for the right and left sensors.

We can now approximate $F(S(\cdot)), F^{\prime}(S(\cdot))$, and $\sigma(S(\cdot))$ around the middle point between the two sensors $(\mathbf{x})$ since $\mathbf{x}_{r}=\mathbf{x}-\frac{\delta}{2} \hat{\mathbf{e}}_{p}$ and $\mathbf{x}_{l}=\mathbf{x}+\frac{\delta}{2} \hat{\mathbf{e}}_{p}$, where $\delta$ is the distance between sensors, and $\hat{\mathbf{e}}_{p}=[-\sin \theta, \cos \theta]$ is the unit vector perpendicular to the vehicle motion direction $(\theta)$ as:

$$
\begin{aligned}
\mathcal{F}\left(S\left(\mathbf{x}_{r}\right)\right) & =\mathcal{F}(S(\mathbf{x}))-\frac{\delta}{2} \mathcal{F}^{\prime}(S(\mathbf{x})) \nabla S(\mathbf{x}) \cdot \hat{\mathbf{e}}_{p} \\
\mathcal{F}\left(S\left(\mathbf{x}_{l}\right)\right) & =\mathcal{F}(S(\mathbf{x}))+\frac{\delta}{2} \mathcal{F}^{\prime}(S(\mathbf{x})) \nabla S(\mathbf{x}) \cdot \hat{\mathbf{e}}_{p}
\end{aligned}
$$

where $\mathcal{F}$ represents any of the composite functions to approximate $F(S(\cdot)), F^{\prime}(S(\cdot))$, and $\sigma(S(\cdot))$. Substituting 
equations (3) into (2), and using the conversion from wheel velocities $\left(v_{r}\right.$ and $\left.v_{l}\right)$ to linear and angular speeds of the robot $(v$ and $\omega=\dot{\theta}$ ) we get:

$$
\begin{aligned}
\omega & =-\frac{\delta}{d} \nabla F(\mathbf{x})^{T} \hat{\mathbf{e}}_{p}-\frac{1}{d} D_{1}(\mathbf{x}, \theta) d W_{t}^{-}-\frac{1}{d} D_{2}(\mathbf{x}, \theta) d W_{t}^{+} \\
v & =F(\mathbf{x})+\frac{1}{2} D_{1}(\mathbf{x}, \theta) d W_{t}^{+}+\frac{1}{2} D_{2}(\mathbf{x}, \theta) d W_{t}^{-}
\end{aligned}
$$

where for simplicity we denote $F(\mathbf{x})=F(S(\mathbf{x})), d$ is the wheelbase of the vehicle, $d W_{t}^{+}=d W_{t}^{r}+d W_{t}^{l}$ and $d W_{t}^{-}=$ $d W_{t}^{l}-d W_{t}^{r}$ are two stochastic processes combining the two sensors noise, and the terms $D_{1}$ and $D_{2}$ are:

$$
\begin{aligned}
& D_{1}(\mathbf{x}, \theta)=\frac{\delta^{2}}{4} F^{\prime \prime}(\mathbf{x}) \sigma(\mathbf{x})\left[\nabla S(\mathbf{x}) \cdot \hat{\mathbf{e}}_{p}\right]^{2}+F^{\prime}(\mathbf{x}) \sigma(\mathbf{x}) \\
& D_{2}(\mathbf{x}, \theta)=\frac{\delta}{2} \nabla S(\mathbf{x}) \cdot \hat{\mathbf{e}}_{p}\left[F^{\prime}(\mathbf{x}) \sigma^{\prime}(\mathbf{x})+F^{\prime \prime}(\mathbf{x}) \sigma(\mathbf{x})\right]
\end{aligned}
$$

where we further eliminated all the compound functions to simplify notation, i.e. $F(\mathbf{x})=F(S(\mathbf{x})), F^{\prime}(\mathbf{x})=F^{\prime}(S(\mathbf{x}))$, and so on.

It is worth noting that the stochastic processes $d W_{t}^{+}$and $d W_{t}^{-}$are not independent random processes, but equations (4) can be easily re-stated in terms of the independent Wiener processes $d W_{t}^{r}$ and $d W_{t}^{l}$. Moreover, it can be shown that $d W_{t}^{+}$and $d W_{t}^{-}$are the increments of two Wiener processes with $W_{t+s}^{+}-W_{t}^{+}$and $W_{t+s}^{-}-W_{t}^{-}$following a Gaussian distribution with variance $2 s$.

Substituting equations (4) into the unicycle motion model we can express the behaviour of Braitenberg vehicle $3 \mathrm{a}$ using Itô calculus as a stochastic differential equation (SDE):

$$
\begin{gathered}
d x_{t}=F\left(\mathbf{x}_{t}\right) \cos \theta_{t} d t+\frac{1}{2} D^{-}\left(\mathbf{x}_{t}, \theta_{t}\right) \cos \theta_{t} d W_{t}^{r} \\
+\frac{1}{2} D^{+}\left(\mathbf{x}_{t}, \theta_{t}\right) \cos \theta_{t} d W_{t}^{l} \\
d y_{t}=F\left(\mathbf{x}_{t}\right) \sin \theta_{t} d t+\frac{1}{2} D^{-}\left(\mathbf{x}_{t}, \theta_{t}\right) \sin \theta_{t} d W_{t}^{r} \\
+\frac{1}{2} D^{+}\left(\mathbf{x}_{t}, \theta_{t}\right) \sin \theta_{t} d W_{t}^{l} \\
d \theta_{t}=-\frac{\delta}{d} \nabla F\left(\mathbf{x}_{t}\right) \cdot \hat{\mathbf{e}}_{p}\left(\theta_{t}\right) d t-\frac{1}{d} D^{+}\left(\mathbf{x}_{t}, \theta_{t}\right) d W_{t}^{l} \\
+\frac{1}{d} D^{-}\left(\mathbf{x}_{t}, \theta_{t}\right) d W_{t}^{r}
\end{gathered}
$$

where $D^{-}\left(\mathbf{x}_{t}, \theta_{t}\right)=D_{1}\left(\mathbf{x}_{t}, \theta_{t}\right)-D_{2}\left(\mathbf{x}_{t}, \theta_{t}\right)$ and $D^{+}\left(\mathbf{x}_{t}, \theta_{t}\right)=D_{1}\left(\mathbf{x}_{t}, \theta_{t}\right)+D_{2}\left(\mathbf{x}_{t}, \theta_{t}\right)$. This is a non-linear drift-diffusion equation with a drift term equivalent to the deterministic model of Braitenberg vehicle $3 a$ [18], and diffusion terms depending on the sensors noise, the connecting function (i.e. the controller), and the gradient of the stimulus, see equations (5). In general, the diffusion term, for arbitrary $F(s)$, will be non-linear even if the sensor noise is additive $\sigma(S(\mathbf{x}))=\sigma_{0}$. Only when the connection function $F(s)$ is linear and the noise is additive, the SDE describing the movement of the vehicle will have an additive noise term, but, as we will see later, it appears only in the equation describing the angular variable. Although an analytic solution for these equations cannot be found in general, as we will show later, this stochastic differential equation can be used to simulate and analyse the movement of the vehicle under sensor noise conditions.

Interestingly, if the vehicle is immersed in a constant stimulus, i.e. $\nabla S(\mathbf{x})=\mathbf{0}$ the heading has no drift term, and the vehicle direction suffers a dispersion with variance growing over time proportional to the sensor noise, i.e. $\sigma_{\theta}^{2} \propto \frac{F^{\prime}\left(s_{0}\right)^{2}}{d^{2}} \sigma\left(s_{0}\right)^{2} t$, where $\sigma(s)$ and the derivative of $F(s)$ are both evaluated at the stimulus value $S(\mathbf{x})=s_{0}$.

\section{EVOLUTION OF THE FIRST TWO MOMENTS}

Let us assume a general stochastic differential equation, like (6) in the Itô form:

$$
\mathbf{d} \mathbf{X}_{t}=\mathbf{F}\left(\mathbf{X}_{t}\right) d t+\mathbf{G}\left(\mathbf{X}_{t}\right) \mathbf{d} \mathbf{W}_{t}
$$

where $\mathbf{X}_{t}$ is an $n$ dimensional state, $\mathbf{F}\left(\mathbf{X}_{t}\right)$ and $\mathbf{G}\left(\mathbf{X}_{t}\right)$ are the nonlinear drift and diffusion terms respectively, and $\mathbf{d} \mathbf{W}_{t}$ is an $m$ dimensional vector of increments of independent Wiener processes. It is worth reminding that the stochastic process is a set of infinite (in this case uncountable) random variables $\mathbf{X}$ indexed by time $\mathbf{X}_{t}$. If we denote the expected value of $\mathbf{X}$ at time $t$ as $E\left[\mathbf{X}_{t}\right]=\boldsymbol{\mu}_{\boldsymbol{t}}$, taking the expectation on both sides of (7) we obtain a differential equation describing the evolution of the average:

$$
\dot{\boldsymbol{\mu}}_{t}=E\left[\mathbf{F}\left(\mathbf{X}_{t}\right)\right]
$$

where the expectation operator $E[]$ is applied to the nonlinear function $F(\cdot)$, and the second term of (7) can be neglected as $\int_{t_{0}}^{t} E\left[\mathbf{G}\left(\mathbf{X}_{t}\right) \mathbf{d} \mathbf{W}_{t}\right]=0$ for the Itô integral. To compute the expectation of a scalar non-linear function we can use the first order approximate around the average $F(\boldsymbol{\mu}+(\mathbf{X}-\boldsymbol{\mu})) \approx F(\boldsymbol{\mu})+D F(\boldsymbol{\mu})(\mathbf{X}-\mu)+\frac{1}{2}(\mathbf{X}-$ $\mu)^{T} D^{2} F(\boldsymbol{\mu})(\mathbf{X}-\mu)$, where $D F(\boldsymbol{\mu})$ and $D^{2} F(\boldsymbol{\mu})$ represent, respectively, the first (gradient) and second (Hessian) derivatives of the function $F(\mathbf{X})$ evaluated at the average. Because $E[D F(\boldsymbol{\mu})(\mathbf{X}-\mu)]=0$, and for a random variable $\mathbf{X}$ with covariance $\Sigma$ and a symmetric matrix $A, E\left[\mathbf{X}^{T} A \mathbf{X}\right]=$ $\operatorname{tr}(A \Sigma)+E[\mathbf{X}]^{T} A E[\mathbf{X}]$, with $\operatorname{tr}()$ denoting the trace of a matrix, the equation for the evolution of the average can be approximated as:

$$
\dot{\boldsymbol{\mu}}_{t}=\mathbf{F}\left(\boldsymbol{\mu}_{t}\right)+\operatorname{Tr}\left(D^{2} \mathbf{F}\left(\boldsymbol{\mu}_{t}\right) \Sigma_{t}\right)
$$

where the term $\operatorname{Tr}\left(D^{2} \mathbf{F}(\boldsymbol{\mu}) \Sigma_{t}\right)$ is a vector containing the traces of the product of the Hessians of the components of $\mathbf{F}(\mathbf{X})=\left(F_{i}(\mathbf{X})\right)$ evaluated at the mean $\boldsymbol{\mu}_{t}$, multiplied by the covariance matrix of $\mathbf{X}_{t}, \Sigma_{t}$. This equation shows that the average of a set of trajectories of this Braitenberg vehicle is not equivalent to the behaviour of the noiseless vehicle, since the second term in (9) is not zero in general.

Although it is more involved, using Itô's lemma, we can compute the time evolution of the covariance matrix (second moment) for the SDE (7) defining a new variable $\mathbf{Y}_{t}=\left(\mathbf{X}_{t}-\boldsymbol{\mu}_{t}\right)\left(\mathbf{X}_{t}-\boldsymbol{\mu}_{t}\right)^{T}$ for which the expectation is the covariance of $\mathbf{X}$, i.e. $E\left[\mathbf{Y}_{t}\right]=\Sigma_{t}$. The dynamical equation for the covariance depends on the Jacobian of the drift term and on the diffusion term (its first and second order derivatives). However, as we will see in the next section, 
under some assumptions the dynamical equation for the covariance matrix can be greatly simplified. Alternatively, the evolution of the moments for (7) can be obtained through the Fokker-Planck equation [5].

\section{A. Simplifying assumptions on the noise}

As we already stated, the stochastic differential equation modelling the movement of Braitenberg vehicle $3 \mathrm{a}$ with noisy sensors, equation (6), becomes linear for the angular variable in the case of additive noise $\left(\sigma(s)=\sigma_{0}\right)$, and linear connecting function $\left(F(s)=a_{0}-a_{1} s\right)$ simultaneously. It is worth reminding (cf. section II) that the function connecting the sensors and the motors has to be decreasing for this vehicle, therefore the slope has to be negative $\left(a_{1}>0\right)$. Under these assumptions the drift terms become $D_{1}(\mathbf{x}, \theta)=a_{1} \sigma_{0}$ and $D_{2}(\mathbf{x}, \theta)=0$, and the stochastic differential equations describing the movement of the Braitenberg vehicle become:

$$
\begin{aligned}
& d x_{t}=F\left(\mathbf{x}_{t}\right) \cos \theta_{t} d t+\frac{a_{1} \sigma_{0}}{2} \cos \theta_{t}\left(d W_{t}^{r}+d W_{t}^{l}\right) \\
& d y_{t}=F\left(\mathbf{x}_{t}\right) \sin \theta_{t} d t+\frac{a_{1} \sigma_{0}}{2} \sin \theta_{t}\left(d W_{t}^{r}+d W_{t}^{l}\right) \\
& d \theta_{t}=-\frac{\delta}{d} \nabla F\left(\mathbf{x}_{t}\right) \cdot \hat{\mathbf{e}}_{p}\left(\theta_{t}\right) d t-\frac{a_{1} \sigma_{0}}{d}\left(d W_{t}^{l}-d W_{t}^{r}\right)
\end{aligned}
$$

As it can be seen, the diffusion terms in the first two equations include the sin and cos of the angular variable, therefore the noise is non additive for the $x$ and $y$ equations. For the heading direction, however, the diffusion factor scaling the noise processes on the left and right sensors is constant. Comparing equation (10) and (7), and defining the state $\mathbf{X}_{t}=\left(\mathbf{x}_{t}, \theta_{t}\right)$ we can identify the drift and diffusion terms as:

$$
\mathbf{F}\left(\mathbf{X}_{t}\right)=\left[\begin{array}{c}
F\left(\mathbf{x}_{t}\right) \cos \theta_{t} \\
F\left(\mathbf{x}_{t}\right) \sin \theta_{t} \\
-\frac{\delta}{d} \nabla F\left(\mathbf{x}_{t}\right) \cdot \hat{\mathbf{e}}_{p}\left(\theta_{t}\right)
\end{array}\right]
$$

and

$$
\mathbf{G}\left(\mathbf{X}_{t}\right)=\left[\begin{array}{cc}
\frac{a_{1} \sigma_{0}}{2} \cos \theta_{t} & \frac{a_{1} \sigma_{0}}{2} \cos \theta_{t} \\
\frac{a_{1} \sigma_{0}}{2} \sin \theta_{t} & \frac{a_{1} \sigma_{0}}{2} \sin \theta_{t} \\
\frac{a_{1} \sigma_{0}}{d} & -\frac{a_{1} \sigma_{0}}{d}
\end{array}\right]
$$

and $d \mathbf{W}_{t}=\left[d W_{t}^{r}, d W_{t}^{l}\right]^{T}$.

Using equation (9) we can obtain the evolution of the average pose of the vehicle as:

$$
\begin{aligned}
& \dot{\mu}_{x}=F\left(\boldsymbol{\mu}_{x y}\right) \cos \mu_{\theta}+\operatorname{tr}\left(H_{x} \Sigma\right) \\
& \dot{\mu}_{y}=F\left(\boldsymbol{\mu}_{x y}\right) \sin \mu_{\theta}+\operatorname{tr}\left(H_{y} \Sigma\right) \\
& \dot{\mu}_{\theta}=-\frac{\delta}{d} \nabla F\left(\boldsymbol{\mu}_{x y}\right) \cdot \hat{\mathbf{e}}_{p}\left(\mu_{\theta}\right)+\operatorname{tr}\left(H_{\theta} \Sigma\right)
\end{aligned}
$$

where $\boldsymbol{\mu}_{x y}=\left[\mu_{x}, \mu_{y}\right]^{T}, \Sigma$ is the covariance matrix, and $H_{x}$, $H_{y}$ and $H_{\theta}$ are the Hessian matrices of the corresponding components of the drift vector flow (11).

Using the technique of defining a new stochastic process $\mathbf{Y}_{t}=\left(\mathbf{X}_{t}-\boldsymbol{\mu}_{t}\right)\left(\mathbf{X}_{t}-\boldsymbol{\mu}_{t}\right)^{T}$, the evolution equation of the covariance under the stated assumptions can be shown to be:

$$
\dot{\Sigma}=D F\left(\boldsymbol{\mu}_{x y}, \mu_{\theta}\right) \Sigma+\Sigma D F\left(\boldsymbol{\mu}_{x y}, \mu_{\theta}\right)^{T}+G(\mathbf{X}) G(\mathbf{X})^{T}
$$

where $D F\left(\boldsymbol{\mu}_{x y}, \mu_{\theta}\right)$ is the Jacobian of the drift flow evaluated at the mean pose of the vehicle.

While the stochastic differential equations (6) can be used to simulate individual trajectories of vehicle $3 \mathrm{a}$ under sensor noise conditions, equations (13) represents the average behaviour of the simulation under multiple noise realisations, while equation (14) corresponds to the covariance matrix of the trajectories. Interestingly, while the average trajectory in the stochastic case includes the term on the covariance matrix of the pose, for the stochastic differential equation the drift term simply corresponds to the noiseless model of Braitenberg vehicle $3 \mathrm{a}$.

\section{B. Vehicle in a stimulus with a constant gradient}

Although having additive noise in the sensors simplifies the equations for the average and variance of the dynamical system (6), no analytical conclusions can be easily drawn from the model behaviour. However, if we further assume the vehicle is immersed in a stimulus with a constant gradient $\nabla S(\mathbf{x})=\nabla_{0}$, and if we focus on the particular case where its heading is aligned with the direction of the gradient, i.e. $\nabla_{0} \cdot \hat{e}_{p}=0$, the dynamical equations for the average and standard deviation can be further simplified and clarifying analytical results can be obtained. For simplicity we will only consider what happens to the angular variable, its mean and variance under these assumptions, as the evolution equations of $x$ and $y$ do not allow to analytically interpret the vehicle's behaviour. The last equation of (13) then becomes:

$$
\dot{\mu}_{\theta}=-\frac{\delta}{d}\left[1-\frac{\sigma_{\theta \theta}}{2}\right] a_{1} \nabla_{0} \cdot \hat{e}_{p}
$$

which vanishes when the vehicle is initially heading the direction of the source $\left(\nabla S(\mathbf{x})=\nabla_{0}\right)$. In the deterministic case, if the vehicle is aligned with the stimulus gradient, its heading direction does not change $(\dot{\theta}=0)$. The average heading of the stochastic equations also fulfils $\dot{\mu}_{\theta}=0$ and therefore the average direction of the vehicle does not change. Because of the additional covariance term, the expected $x$ and $y$ coordinates do not necessarily behave as the deterministic case. Applying the simplifying conditions to equation (14), and taking only the variance of the angular velocity, the dispersion around the direction of the gradient $\sigma_{\theta \theta}$ is found to follow the equation:

$$
\dot{\sigma}_{\theta \theta}=2 \frac{a_{1}^{2} \sigma_{0}^{2}}{d^{2}}+2 \frac{\delta}{d} a_{1} \nabla_{0} \cdot \hat{e} \sigma_{\theta \theta}
$$

where $\hat{e}=[\cos \theta, \sin \theta]^{T}$. This is a linear differential equation on the variance $\sigma_{\theta \theta}$, and it is worth noting that the equation generalises our previous results on the dispersion of the heading direction in the presence of a constant stimulus $\left(\nabla S(\mathbf{x})=\mathbf{0}\right.$, see $\sigma_{\theta}^{2}$ in section II). The stability of this equation depends on the dot product of the gradient and the unit direction of the vehicle $\hat{e}$ evaluated at the expected heading $\mu_{\theta}$. If the vehicle is heading the direction in which the stimulus grows, i.e. $\nabla_{0} \cdot \hat{e}=\left|\nabla_{0}\right|$, where $|\cdot|$ represents the norm of the vector, the differential equation (16) is unstable and the variance of the heading direction grows exponentially. This result is easy to understand intuitively, 
since vehicle 3 a performs a gradient descent on the stimulus $S(\mathrm{x})$ with non-holonomic constraints. When the vehicle approximately heads the direction in which the stimulus grows (but not the exact gradient direction) it turns to face the opposite direction. That occurs for the deterministic case, and the corresponding stochastic behaviour is an exponential growth in the dispersion, although on average the vehicle does not turn, which corresponds to the alignment with the gradient in the deterministic case. However, when the initial direction of the vehicle is opposite to the gradient, the dot product is negative, $\nabla_{0} \cdot \hat{e}=-\left|\nabla_{0}\right|$, and the differential equation above is stable. The equation can be solved analytically leading to the typical time exponential decay term in the transient with time constant $\tau^{-1}=2 \frac{\delta}{d} a_{1}\left|\nabla_{0}\right|$. Because the equation is stable, the equilibrium point in this case can be obtained by simply making $\dot{\sigma}_{\theta \theta}=0$ and solving for the variance $\sigma_{\theta \theta}$, which turns to be:

$$
\sigma_{\theta \theta}=\frac{a_{1} \sigma_{0}^{2}}{d \delta\left|\nabla_{0}\right|}
$$

This is an interesting result as it provides a boundary for the angular dispersion of the non-holonomic vehicle direction when performing a gradient descent, and can be intuitively interpreted to some extent. Obviously, the higher the noise variance in the sensors $\sigma_{0}^{2}$, the higher the angular dispersion of the vehicle will be. On the other hand, if the gradient is very steep, i.e. $\left|\nabla_{0}\right|$ is large, the angular variance decreases. Interestingly $\sigma_{\theta \theta}$ in the equilibrium increases linearly with the slope of the connection function $a_{1}$, which affects the stability in the deterministic case, i.e. how fast the vehicle turns towards the decreasing stimulus direction. This implies that there is a trade-off between the capacity of the vehicle to reach the target and the heading dispersion. If the connection function $F(s)$ has large negative slope it will help the vehicle to reach the target in the absence of noise. However, the slope will contribute to a more erratic heading direction under sensor noise condition.

\section{Simulations}

This section will present a set of simulations under different conditions for the stochastic differential equations modelling Braitenberg vehicle 3a. While the stochastic differential equations are integrated in all the simulations using the Euler-Maruyama method with step $h=0.01$ time units, their deterministic counterparts are solved using the standard Runge-Kutta methods of order 4-5 and adaptive step size.

\section{A. Additive vs. multiplicative sensor noise}

As stated above, even in the case of additive sensor noise the final approximated equations show multiplicative noise in $x$ and $y$, but simulations can provide an idea of the vehicle behaviour. Figures 2(a) and 2(b) show the results of simulating the equations (6) under two different noise conditions for 500 different realisations of the process noise. The average trajectory is shown in the figure as a dashed line, jointly with the solution of the noiseless system, i.e. the corresponding deterministic equation. All the simulations

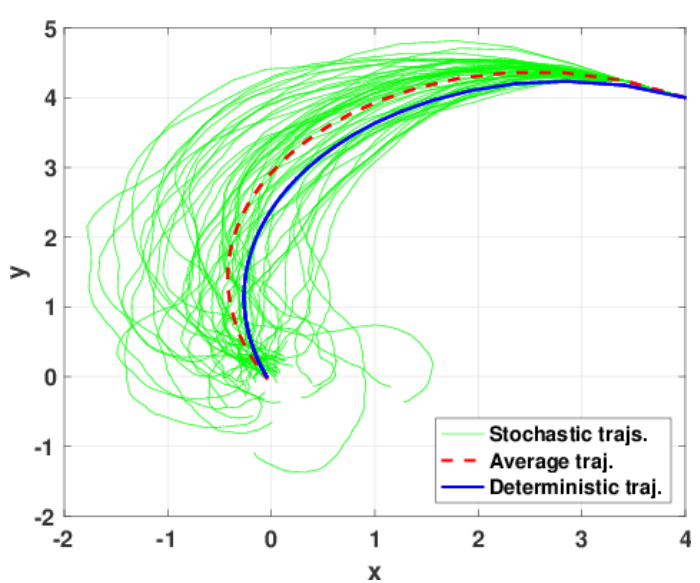

(a) Additive noise

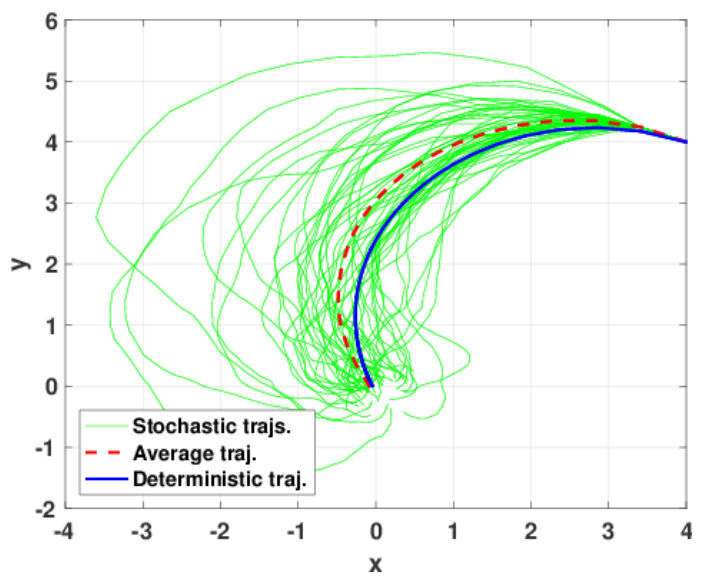

(b) Multiplicative noise

Fig. 2. Stochastic model of Braitenberg vehicle 3 a with additive and nonadditive noise.

have the same stimulus function and initial pose of the robot. As the model predicts, although the drift term is the same as the deterministic model, the equation for the time evolution of the average trajectory, equation (13), differs from the deterministic case. The figures clearly show that the average trajectory of the vehicles with noise is longer than the noiseless vehicles. This is captured by the additional terms on the equations of the average trajectories (13), which depend on the Hessian of the stimulus and the covariance matrix. Because the Hessian is related to the curvature of the isolines of $S(\mathbf{x})$, whether the average trajectory is longer or shorter than the deterministic case might depend on the local curvature of the stimulus close to the deterministic trajectory. A comparison of figures 2(a) and 2(b) also shows that the deviation from the average (the trajectory covariance) is larger in the case of non-additive noise. This occurs despite the fact that the noise variance in the non-additive simulations goes to zero as the vehicle approaches the target (i.e. the signal to noise ratio goes to infinity), and the variance of the non-additive case was smaller than the additive one. 

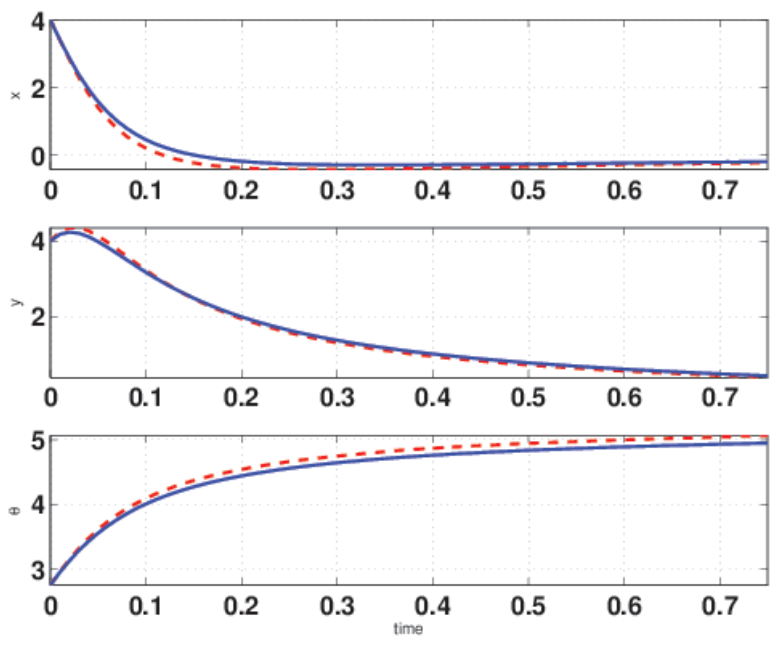

(a) Average trajectory
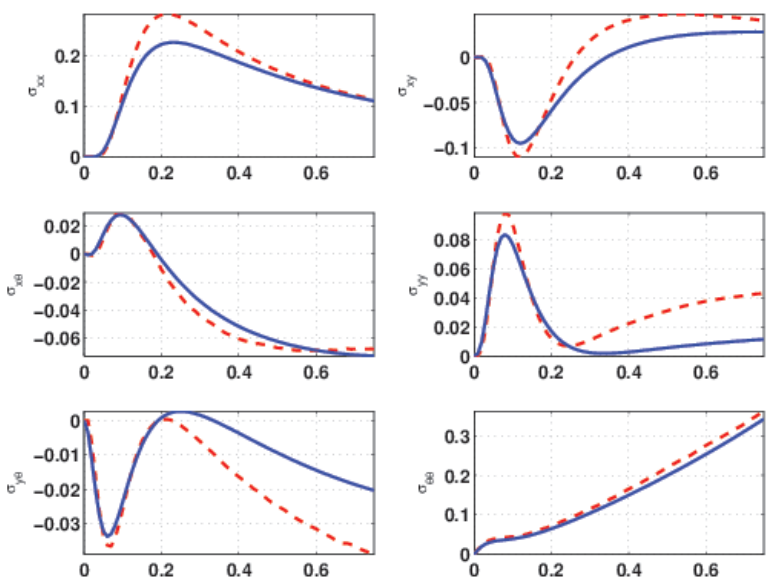

(b) Covariance trajectory

Fig. 3. Average and covariance matrix from SDE and moments equation.

\section{B. Evolution of the first two moments}

We also performed a comparison between the results from simulating the stochastic differential equations (6) for the additive noise case, and the evolution equations of the average trajectory and its corresponding covariance matrix, equations (13) and (14). It is worth noting that these last two equations are vector and matrix differential equations which have to be integrated simultaneously, as the computation of the mean requires the covariance matrix. Figures 3(a) and 3(b) show the time evolution of the mean trajectories and the entries of the covariance matrix. The solid line represents the evolution obtained from the deterministic evolution equations of the moments, while the dashed line has been computed from the simulation of 15000 trajectories for different realisations of the sensor noise. Figure 3(a) shows a disparity between the average trajectories obtained from the two sets of equations, which can be seen more clearly in the evolution of the covariance matrix (figure 3(b)). This results from considering only the first two moments, jointly with the approximation of the expectations as a Taylor series. As can be seen in figure $3(\mathrm{~b})$ the elements of the covariance matrix obtained through equation (14) - solid line - start close to the covariance of the simulated trajectories (dashed line). However, as time unfolds they start to diverge, as the approximation errors accumulate through the integration. Because the dynamical system is non linear and the final noise is non additive, although the original noise is Gaussian, the pose at each point in time is not, as Gaussianity test run on the sampled trajectories confirmed.

\section{Behaviour in a constant gradient stimulus}

To test the validity of the heading deviation variance, equation (17), in a constant gradient stimulus we performed simulations and compared the results of additive and nonadditive noise. Figures 4(a) and 4(b) show simulations of 500 different realisations of the noise for a vehicle $3 \mathrm{a}$ immersed in a stimulus with a constant gradient with its initial orientation aligned in the opposite direction to the gradient. According to the results obtained in section III-B the variance of the heading direction goes to a finite value as time goes to infinity in the case of additive noise. Figure 4(a) presents the corresponding simulations where the dashed lines provide a $3 \sigma$ boundary around the average angular trajectory according to the analytical result. The red dashed line represents the actual $3 \sigma$ boundary for the trajectories obtained through simulation of equation (6). As it can be seen there is a growing discrepancy between the two plots due to a few trajectories with angular values around $\pm 6 \mathrm{rad}$, which is actually the same orientation as $0 \mathrm{rad}$, but the angle has not been normalised to allow modelling the angle as Gaussian distribution (although a von Misses distribution would be more appropriate). Therefore the vehicles following these trajectories found an equivalent attractor to the expected average, after making a whole turn due to the noise. It is expected that the experimental variance will increase over time as other simulated trajectories might find the other equivalent attractors $\pm 2 k \pi$, with $k=2,3, \cdots$. In the case at hand, the angular variance has a short time constant as the plot shows, since the variance reaches the steady state regime in less than one simulated time unit. Figure 4(b) shows a similar simulation set up with a non additive noise, where the analytic approximation does not work. These conditions are not captured by our assumptions, therefore further analysis would be required to understand the large changes in the direction of the vehicle, but this is out of the scope of the current work.

\section{CONCLUSIONS AND FUTURE WORK}

This paper presents the first drift-diffusion model of Braitenberg vehicle $3 \mathrm{a}$. While on the one hand existing theoretical models assumed noise free sensors, on the other hand, many experimental research results have shown this bio-inspired source seeking mechanism works with noisy sensors. While it is well known that adding a random vector field to the gradient of a potential field contributes to avoid local minima, in a recent work [17] a drift diffusion approach with a similar spirit is presented, adding random noise to the navigation function itself. The resulting model for the robot 


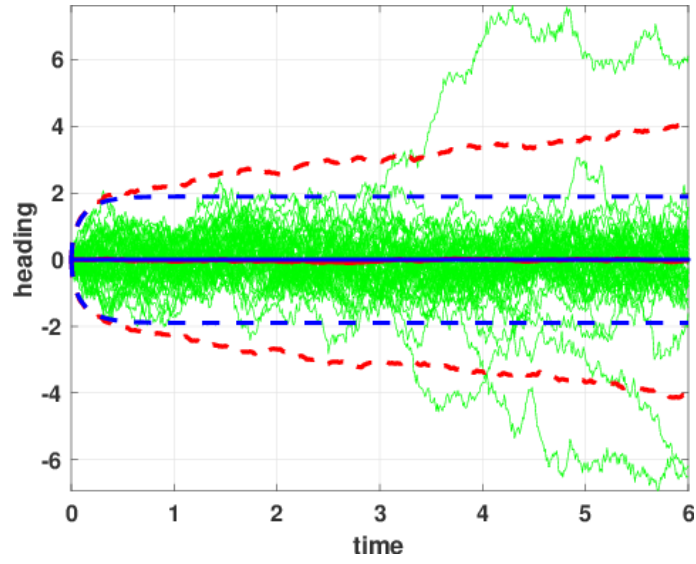

(a) Additive noise

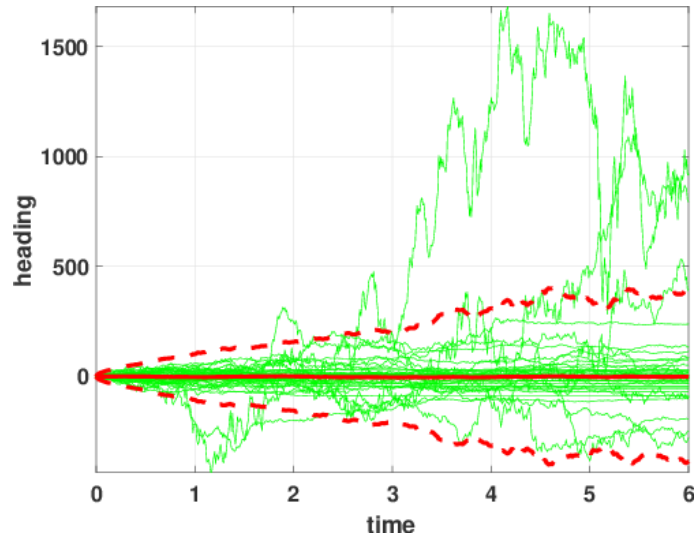

(b) Multiplicative noise

Fig. 4. Heading directions of vehicle $3 \mathrm{~b}$ in a stimulus with constant gradient.

motion is a drift-diffusion equation which allows obtaining some theoretical results on the probability of avoiding local minima. The noise signal, however, also affects the ability of the robot to reach the target. In this work, we explicitly tackled this issue by proposing the first stochastic formulation of target reaching with Braitenberg vehicles, and showed that the analytical solution is a drift-diffusion process. Our analytic results show that the expression of the variance on the angular variable provides an excellent clue on whether the robot is deviating too much from the target over time. Finding a bound of the position of the robot around the goal is also useful to analytically decide when the target has been reached. In the case of this non-holonomic controller complete analytical resolution of the system is not possible unless further assumptions are made whose consequences we illustrate through numerical simulations.

Besides providing a mechanism to perform realistic simulations of Braitenberg vehicle $3 \mathrm{a}$, the proposed model can serve as a basis to implement both model-based (e.g. metric or topological maps) and model-free (sensor based reactive) implementations of bio-inspired navigation strategies in robotics. Our future plans include to investigate the interplay between these two strategies [19] to implement a biologically inspired robot endowed with navigation learning capabilities. This entails strategically combining potential functions representing goals in the environment and stimulus functions based on direct perception from the robot. To do so, however, other navigation primitives might need to be used, specifically avoidance strategies like Braitenberg vehicle $2 \mathrm{a}$.

\section{ACKNOWLEDGEMENTS}

This work was supported by the Royal Society International Exchange Scheme under grant IE151293. KW-L and IR were supported by the ASUR Programme under grant 1014-C4-Ph1-456 071.

\section{REFERENCES}

[1] V. Braitenberg, Vehicles. Experiments in synthetic psychology. The MIT Press, 1984.

[2] I. Rañó, "Biologically inspired navigation primitives," Robotics and Autonomous Systems, vol. 62, no. 10, pp. 1361-1370, 2014.

[3] G. Arechavaleta, J. P. Laumond, H. Hicheur, and A. Berthoz, "An optimality principle governing human walking," IEEE Transactions on Robotics, vol. 24, no. 1, pp. 5-14, 2008.

[4] I. Rañó, "The bio-inspired chaotic robot," in Proc. of the IEEE International Conference on Robotics and Automation, 2014, pp. 304 309.

[5] N. V. Kampen, Stochastic processes in physics and chemistry. Elsevier, 1992.

[6] X. Zhou, K. Wong-Lin, and P. Holmes, "Time-varying perturbations can distinguish among integrate-to-threshold models for perceptual decision making in reaction time tasks," Neural Computation, vol. 21 , p. 23362362, 2009.

[7] E. Codling, M. Plank, and S. Benhamou, "Random walks in biology," Journal of the Royal Society Interface, vol. 5, pp. 813-834, 2008.

[8] B. Webb, A Spiking Neuron Controller for Robot Phonotaxis. The MIT/AAAI Press, 2001, pp. 3-20.

[9] A. Horchler, R. Reeve, B. Webb, and R. Quinn, "Robot phonotaxis in the wild: a biologically inspired approach to outdoor sound localization," Advanced Robotics, vol. 18, no. 8, pp. 801-816, 2004.

[10] R. Reeve, B. Webb, A. Horchlerb, G. Indiveri, and R. Quinn, "New technologies for testing a model of cricket phonotaxis on an outdoor robot," Robotics and Autonomous Systems, vol. 51, no. 1, pp. 41-54, 2005

[11] M. Bernard, S. N'Guyen, P. Pirim, B. Gas, and J.-A. Meyer, "Phonotaxis behavior in the artificial rat psikharpax," in International Symposium on Robotics and Intelligent Sensors, IRIS2010, 2010.

[12] D. Shaikh, J. Hallam, J. Christensen-Dalsgaard, and L. Zhang, "A Braitenberg lizard: Continuous phonotaxis with a lizard ear model," in Proceedings of the 3rd International Work-Conference on The Interplay Between Natural and Artificial Computation, 2009, pp. 439448.

[13] E. Bicho and G. Schöner, "The dynamic approach to autonomous robotics demonstrated on a low-level vehicle platform," Robotics and Autonomous Systems, vol. 21, pp. 23-35, 1997.

[14] A. J. Lilienthal and T. Duckett, "Experimental analysis of gas-sensitive Braitenberg vehicles," Advanced Robotics, vol. 18, no. 8, pp. 817-834, 2004

[15] T. Salumäe, I. Rañó, O. Akanyeti, and M. Kruusmaa, "Against the flow: A braitenberg controller for a fish robot," in Proceedings of the International Conference on Robotics and Automation (ICRA), 2012 , pp. 4210-4215.

[16] V. Lebastard, F. Boyer, C. Chevallereau, and N. Servagent, "Underwater electro-navigation in the dark," in Proceedings of the International Conference on Robotics and Automation (ICRA), 2012, pp. 11551160.

[17] P. Reverdy, B. D. Ilhan, and D. Koditschek, "A drift-diffusion model for robotic obstacle avoidance," in Proceedings of the IEEE/RJS International Conference on Intelligent Robots and Systems, 2015, pp. 6113-6120.

[18] I. Rañó, "A steering taxis model and the qualitative analysis of its trajectories," Adaptive Behavior, vol. 17, no. 3, pp. 197-211, 2009.

[19] M. Khamassi and M. Humphries, "Integrating cortico-limbic-basal ganglia architectures for learning model-based and model-free navigation strategies," Frontiers in Behavioral Neuroscience, vol. 6, 2012 